\title{
Sistema de formación continua para la alfabetización digital de los docentes de la escuela de hotelería y turismo de Cienfuegos
}

\section{Continuing Education System for Digital Literacy of Teachers at Tourism and Hospitality School}

\author{
Raidell Avello Martínez, \\ Universidad de Cienfuegos, Cuba. \\ ravello@ucf.edu.cu \\ Raúl López Fernández \\ Universidad Metropolitana de Ecuador, Ecuador. \\ rlopez@ucf.edu.cu \\ Raúl Alpizar Fernández \\ Universidad de Cienfuegos, Cuba. \\ rafdez@ucf.edu.cu
}

\begin{abstract}
Resumen
A partir de las trasformaciones que las TIC han provocado en todas las esferas de la sociedad, se le ha atribuido gran importancia a la alfabetización digital de los docentes de las escuelas de hotelería y turismo, tanto internacionalmente como en nuestro territorio. Sin embargo, esta formación, no se ha abordado de manera reiterada y sistémica, o sea, se han planificado cursos y entrenamientos, pero aislados y desconectados, además, sin tener en cuenta posibilidades de desarrollo como el aprendizaje colaborativo entre los docentes. El objetivo del presente trabajo es elaborar un sistema de superación continua para la alfabetización digital de los docentes, que tome como base estas complejidades, de modo que el profesor pueda recibir una formación permanente, organizada y con un enfoque colaborativo, acorde con el acelerado avance de las TIC. Este sistema fue valorado por 14 expertos, los cuales lo encontraron adecuado para cumplir con su propósito. Además, comentamos una de las acciones formativas realizada, la tutoría colaborativa, y por último se valora la implementación del sistema hasta el 2015, donde resalta el buen criterio de los docentes con respecto a la organización y su puesta en práctica.
\end{abstract}

\section{Palabras clave}

Formación de docentes en TIC, alfabetización digital, TIC, Hotelería y Turismo.

\begin{abstract}
From the transformations that ICT has caused in all spheres of society, great importance has been attributed to the digital literacy of teachers in schools of hospitality and tourism, both internationally and in our territory. However, this formation has not been addressed repeatedly and systemically, that is, courses and training are planned, but isolated and disconnected, without considering development possibilities such as collaborative learning among teachers. The aim of this work is to develop a continuous improvement system for the digital literacy of teachers, so that the teacher can receive continuous organized training, with a collaborative approach, in line with the rapid advancement of ICT. This system was evaluated by 14 experts who found it suitable to fulfill its purpose. In addition, we discuss one of the training activities undertaken, collaborative mentoring. Finally, the
\end{abstract}


implementation of the system until 2015 is assessed with highlighs on the good judgment of teachers as regards organization and implementation.

Key words

Teacher training in ICT, digital literacy, ICT, Hospitality and Tourism

\section{Introducción}

Las escuelas de hotelería y turismo (EHT) cubanas, surgen a partir de la necesidad de formar y preparar profesionales para el sector turístico (incluyendo en la actualidad: formación profesional, capacitación de trabajadores, Licenciatura en Turismo y postgrado). Al transcurrir los años se fue perfeccionando este sistema hasta crearse en 1991, el Sistema Nacional de Formación Profesional para el Turismo denominado FORMATUR, que responde a la necesidad de tener un centro nacional coordinador y regulador del proceso superior de formación, actualización y reciclaje del personal que labora en la hotelería, la restauración y el turismo. Su importancia está dada por su contribución de asegurar el nivel de capacidad para el desarrollo, la calidad del servicio y la eficiencia del sector, acorde con los estándares internacionales y un alto compromiso con el modelo político (Novoa, 2006).

El claustro de docentes de las EHT es bien heterogéneo, compuesto por ingenieros y licenciados de diferentes especialidades que tienen necesidades particulares en relación con las TIC, como idiomas, gestión turística, marketing, gastronomía, dirección, sistemas de gestión hotelera, etc., además, como es lógico, el rango de edad es amplio, lo cual en ocasiones se manifiesta en un nivel de preparación en las tecnologías de la información y las comunicaciones (TIC) bien diferente.

En este sentido, la celeridad del desarrollo de las significa un reto para la alfabetización digital del docente y su adaptación al proceso educativo. En efecto, el avance de las TIC es superior al período de adaptación a ellas (Area, 2009; Gutiérrez, 2008; Salinas, de Benito, y Lizana, 2014). De esta manera, mientras los docentes todavía no se han adaptado a una tecnología dada, han surgido en paralelo una gran variedad de herramientas con funcionalidades diferenciadas en la manera de comunicarse e interactuar. Esta complejidad dificulta la planificación, estructuración e implementación de la alfabetización digital de los docentes de las EHT cubanas.

De igual modo, es importante destacar que la alfabetización digital de los docentes, de manera general, no se ha abordado con un carácter sistémico y reiterado, o sea, se han planificado cursos y entrenamientos, hacia competencias TIC, pero aislados y desconectados, además, no incluyen otras posibilidades como el aprendizaje colaborativo entre los docentes para el desarrollo de estas competencias.

Por otra parte, se ha apreciado en el contexto internacional, que la formación de los docentes de las EHT solo se dirige a la formación de la especialidad, (i.e. cocina, camarera, idioma, etc.) y en menor medida en los aspectos de las TIC, pedagogía, valores, etc. que se dejan a la espontaneidad de los docentes y a través de terceras

Sistema de formación continua para la alfabetización digital de los docentes de las escuelas de hotelería y turismo cubanas. Raidell Avello, Raúl López y Raúl Alpizar.

Página 2 de 13 
instituciones educativas, lo cual repercute en una formación descontextualizada y poco pertinente al área del turismo.

Todos estos elementos han complejizado la alfabetización digital de los docentes y, a pesar de las iniciativas, es insuficiente el nivel alcanzado. Esto se evidenció en diagnóstico realizado en la EHT de Cienfuegos, utilizando el marco de competencias TIC como referente (Avello y López, 2015), en el que se constató que los docentes tienen bajo dominio de las competencias evaluadas. Es importante destacar que en la última dimensión, desarrollo investigativo - profesional, fue donde se encontraron los resultados más bajos en la investigación (Avello, López, Gómez, Espinosa, y Vázquez, 2014).

Por todas estas razones, el objetivo del presente trabajo es elaborar un sistema de superación continua que permita enfrentar esta situación de manera sistémica que tenga en cuenta las complejidades planteadas, de modo que el profesor pueda recibir una formación permanente, organizada en subsistemas y componentes, colaborativa, de manera cíclica, acorde con el acelerado avance de las TIC.

\section{Objetivo del sistema}

Que los profesores de las EHT logren un nivel de alfabetización digital que satisfaga las competencias requeridas para un adecuado desempeño, en función de los retos que la docencia, la investigación y el sector turístico presentan; todo bajo un proceso de formación continuo, actualizado en su contenido y con un enfoque colaborativo.

\section{Representación gráfica del sistema}

La representación gráfica del sistema se puede observar en la figura 1, donde se expresan los 3 subsistemas del sistema: subsistema de entrada, subsistema de formación continua y subsistema de evaluación. Las relaciones se expresan con flechas azules, y se puede apreciar que existen entre componentes de los subsistemas y entre los subsistemas, estos componentes y relaciones serán explicados en detalles en el siguiente apartado.

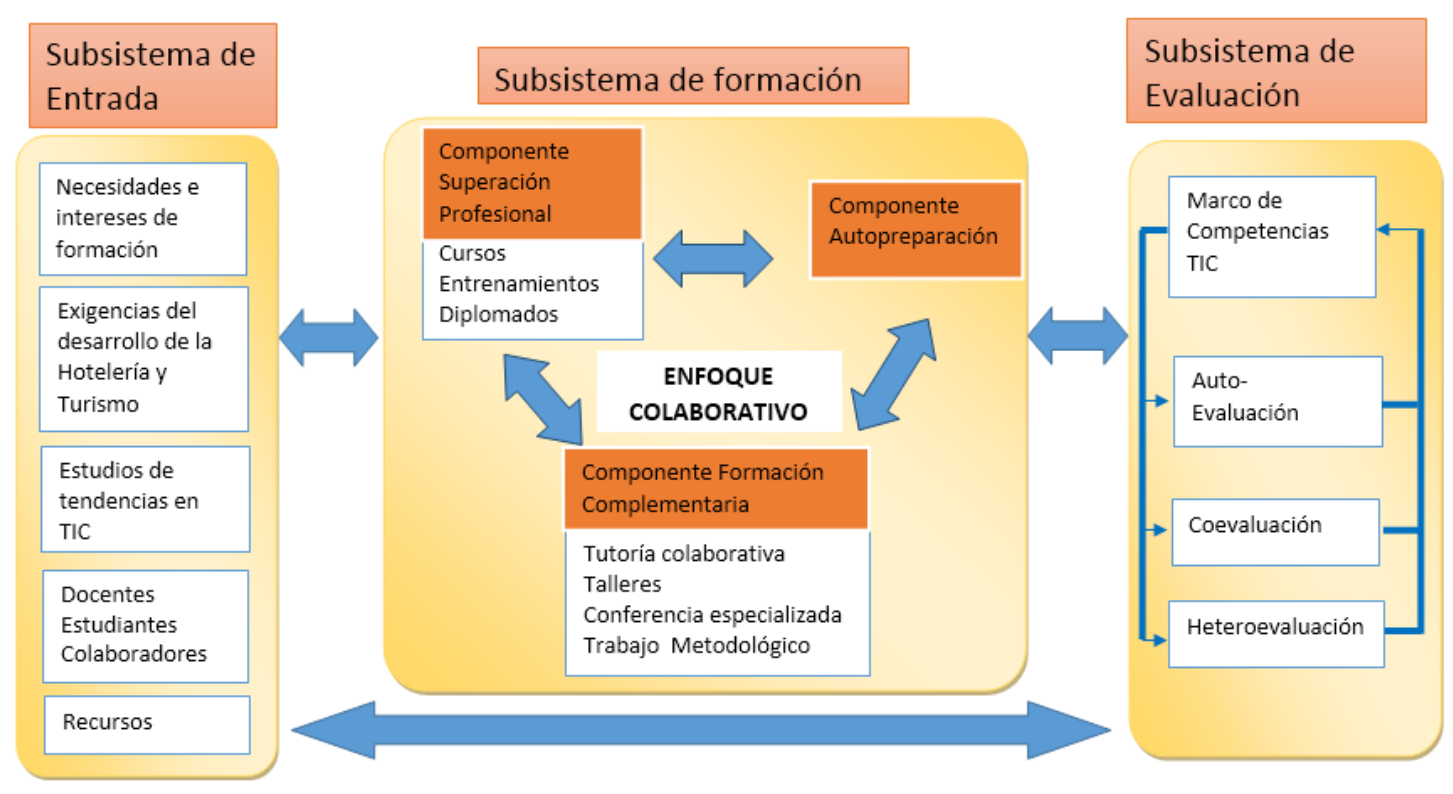

Sistema de formación continua para la alfabetización digital de los docentes de las escuelas de hotelería y turismo cubanas. Raidell Avello, Raúl López y Raúl Alpizar.

Página 3 de 13 
Figura 1. Representación gráfica del sistema de formación continua en alfabetización digital para los docentes de las EHT cubanas. Fuente: Elaboración propia.

\section{Funcionalidad del sistema. Estructura y relaciones}

El subsistema de entrada incluye a los docentes de las EHT, sus necesidades e intereses específicos de formación; las exigencias del desarrollo de la hotelería y turismo; los estudios de tendencias en las TIC y los recursos humanos (profesores, estudiantes y colaboradores), materiales y financieros que aseguran el éxito del proceso de formación. Este subsistema pretende identificar a los docentes con las potencialidades de preparación que tienen para realizar esta tarea; reconocerán las potencialidades curriculares para insertar los conocimientos; incluirán la reflexión al proceso de la actualización respecto a los retos contemporáneos del desarrollo de la hotelería y el turismo, de la formación de los docentes, las estrategias de aprendizaje y sus presupuestos teóricos. Se consideran, además, el proceso acelerado de cambios en las TIC y los recursos necesarios para asegurar el éxito de la formación.

El subsistema de formación se compone de tres componentes: en el primero se agrupan las principales formas de superación que se utilizarán para la alfabetización digital de los docentes. Se reconocen como formas organizativas principales de superación profesional las propuestas en el Reglamento de Postgrado del Ministerio de Educación Superior (2004), que se utilizan como una combinación sistémica, coherente: el curso, para preparar técnica, teórica y metodológicamente a los docentes con respecto al problema a solucionar, complementa o actualiza los conocimientos de los profesionales del sector; el diplomado permite articular los conocimientos adquiridos o actualizados en cursos, conferencias y lograr su integración que propicie la formación de las competencias TIC necesarias; el entrenamiento posibilita la adquisición de habilidades y destrezas y la asimilación e introducción de nuevos procedimientos y tecnologías con el propósito de complementar, actualizar, perfeccionar y consolidar conocimientos y habilidades prácticas en el uso de las TIC.

Se consideró como otro componente la autopreparación, para que los docentes profundicen en los temas abordados y sean capaces de implementar las TIC en sus actividades con la utilización de los recursos y servicios que ofrecen las EHT a través de sus redes.

Como tercer componente se agruparon las tutorías y talleres que se conciben como acciones colaborativas entre los docentes para alcanzar competencias a través de la asesoría directa de docentes aventajados en algunas áreas. Se conciben además las conferencias especializadas y las actividades metodológicas departamentales en las que se pretende socializar las experiencias que tengan los docentes, sobre todo en la puesta en práctica de las competencias de la dimensión pedagógica.

El Subsistema de evaluación contiene al marco de competencias TIC propuesto por Avello y López (2015). Un primer elemento importante es la autoevaluación, que tendrá en cuenta las competencias seleccionadas y utilizarlas como referencia para mantenerse en formación y alcanzar el nivel deseado por la institución; la coevaluación es la evaluación que realizarán los docentes entre ellos mismos; y la heteroevaluación que

Sistema de formación continua para la alfabetización digital de los docentes de las escuelas de hotelería y turismo cubanas. Raidell Avello, Raúl López y Raúl Alpizar.

Página 4 de 13 
realiza la dirección de la escuela o el sistema de escuelas en las visitas y controles, que también tendrá su interacción con el marco de competencias TIC. Este, además de servirle de referencia, puede ser enriquecido en la práctica, agregando nuevas competencias que exijan los cambios en el sector o la sociedad, o eliminando aquellas que resulten obsoletas e integradas en otras. Evidentemente, de esta evaluación surgen nuevas necesidades y es aquí donde se regresa al subsistema de entrada expresando la relación existente entre estos componentes.

El enfoque colaborativo que se persigue en el sistema se refiere a la aplicación de la colaboración entre los docentes en todos los componentes de formación, puesto que la colaboración en el posgrado juega un rol muy importante, porque permite el amplio intercambio de ideas y reflexiones sobre un mismo problema, y se aprovecha gran parte de la experiencia acumulada por los docentes que participan en esta formación.

\section{Formas de instrumentación}

El sistema incluye en su diseño la manera en que se logra implementar en la práctica. Esta posición supera los aspectos logísticos para otorgar prioridad a los procedimientos que guiarán a los implicados en el diseño y desarrollo de los programas, autopreparación y colaboración entre los docentes, tomando como referente el sistema. En este caso, se asume como exigencias:

- La identificación de los sujetos que participan está determinada por la evaluación de sus competencias TIC a través del subsistema de evaluación, como se explicó en el apartado anterior, lo cual supone el tipo de forma de superación que recibe o si lo que requiere es solamente una autopreparación o tutoría.

- La contextualización los contenidos a abordar en las formas de superación según el nivel de aplicación en las esferas de actuación de los docentes de las EHT. Esto se asume como condición para secuenciar estas desde aquellas que precisen mayor o menor tiempo o especialización en el dominio de las competencias propuestas.

- $\quad$ El diseño de programas y materiales necesarios debe revelar la transformación deseada y su efecto en el uso inteligente de las TIC, teniendo en cuenta la fase de diseño de tareas, su desarrollo o evaluación. Así, las conferencias especializadas, la autopreparación y tutorías, etc., pueden generar la elaboración de las actividades docentes, investigativas, laborales o extensionistas que se incluirán en el currículo.

- Explotar al máximo las oportunidades que ofrecen otros espacios y servicios de apoyo a los propósitos de la alfabetización digital, lo cual desde el punto de vista metodológico supone establecer convenios con otros centros y personas. En este sentido, se establecerán convenios con centros universitarios que oferten cursos sobre temas que estén vinculados al desarrollo de las competencias TIC propuestas. Se incluyen además -si fuese necesario- otros docentes especialistas en didáctica que puedan ampliar y profundizar en los temas correspondientes.

- Utilizar las interacciones comunicativas que tienen lugar en la actividad formal o informal, de carácter afectivo, cognitivo o axiológico que se vinculan a esta como

Sistema de formación continua para la alfabetización digital de los docentes de las escuelas de hotelería y turismo cubanas. Raidell Avello, Raúl López y Raúl Alpizar.

Página 5 de 13 
recursos de apoyo psicológico y que pueden influir de manera positiva en los resultados de la formación.

- El entrenamiento juega un rol importante en el sistema, dado por su propia concepción, que exige la integración de la teoría y la práctica, posibilita y exige la demostración, en el desempeño de los docentes, de competencias en el uso adecuado de las TIC y el enfoque colaborativo está presente durante todo el proceso de desarrollo de esta forma de superación profesional.

Asegurado el proceso desde el ámbito metodológico, la ejecución de las formas y programas de superación se realizarán según lo planificado. Serán impartidas por los docentes que han elaborado los programas, a saber: docentes de Informática y docentes aventajados en el dominio de las TIC en relación con la temática de las especialidades o especialistas en temas psicopedagógicos, didactas e investigadores de temas de aplicación de las TIC.

\section{Valoración de los expertos a través de la aplicación del método "Ábaco de Régnier"}

El Ábaco de Régnier es un método de consulta a expertos, concebido por el Doctor François Régnier, con el fin de interrogar a estos y tratar sus respuestas en tiempo real o por vía postal a partir de una escala de colores. Esta técnica utiliza una codificación colorimétrica para expresar esas opiniones (Godet y Durante, 2007). Como todos los métodos de expertos, está destinado a reducir la incertidumbre, confrontar el punto de vista de un grupo con el de otros grupos y a la vez, tomar conciencia de la mayor o menor variedad de opiniones.

La ventaja de este método es que permite efectuar un rápido análisis y de manera didáctica el tratamiento de un problema, facilitando la intercomunicación y el debate en los grupos de trabajo, aunque en primera instancia no se busca el consenso, sino más bien el intercambio de ideas (Ortega y Bracho, 2008).

El método parte de una lógica muy elemental, utiliza los tres colores del semáforo (verde, amarillo y rojo) completados con el verde claro, el rojo claro (permitiendo de este modo suavizar las opiniones). El blanco permite el voto en blanco y el negro la abstención. En él, la escala ordinal se sustituye por una de decisión coloreada que también es ordinal.

En la investigación que se está abordando tiene su aplicación con el fin de que un grupo de expertos, catorce, manifiesten la opinión que poseen sobre el sistema de formación continua en alfabetización digital para los docentes de las EHT.

El método de Ábaco de Régnier se desarrolló teniendo como metodología, las siguientes fases (Crespo, 2009; López, 2009):

\section{Fase 1: Recoger opinión de los expertos}

En esta fase se define, con mayor precisión, la problemática a estudiar, la que será abordada con cuidado y descomponiéndola en elementos (o ítems). Estos ítems serán afirmaciones muy concretas para facilitar el juicio que darán los expertos. Este aspecto

Sistema de formación continua para la alfabetización digital de los docentes de las escuelas de hotelería y turismo cubanas. Raidell Avello, Raúl López y Raúl Alpizar.

Página 6 de 13 
es muy importante, pues si se suministra información ambigua al experto, se corre el riesgo de introducir un grave sesgo en la investigación. Todo esto debe permitir extender el campo de discusión, sobre la evolución del pasado y/o sobre la visión de futuro (Crespo, 2009).

Para la recolección de los datos, se les envió el cuestionario en el cual se explica el objetivo que se persigue con la aplicación de este instrumento y se plantean 5 preguntas:

Pregunta 1: ¿Cómo evalúa usted la estructura del sistema?

Pregunta 2: ¿Qué valoración tiene sobre los subsistemas?

Pregunta 3: ¿Cómo considera la distribución de los componentes en cada subsistema?

Pregunta 4: ¿Cómo considera el enfoque colaborativo que se persigue con el sistema?

Pregunta 5: ¿Posibilidad de emplearlo en otros contextos?

\section{Fase 2: Tratamiento de los datos}

Los datos de las respuestas de los expertos fueron organizados y tabulados, este proceso quedó consolidado en un cuadro colorimétrico, como se observa en la tabla 1.

Tabla 1. Valoración colorimétrica de las respuestas según consulta a expertos por el Ábaco de Régnier

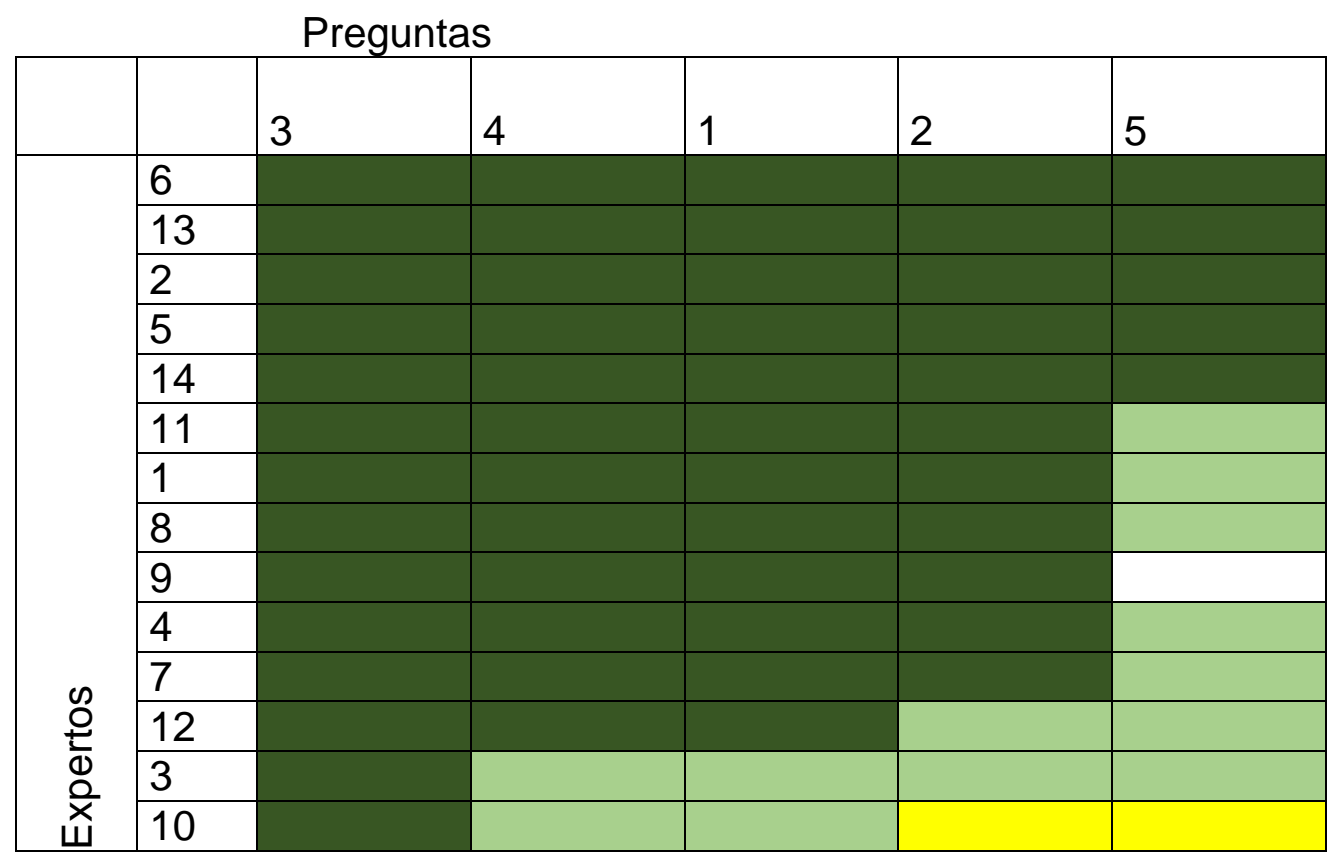

\section{Fase 3: Discusión de los resultados}

Como se puede observar en el cuadro colorimétrico (tabla 1), derivado de las respuestas de los expertos, que existen cuatro colores, coincidiendo, la mayoría, con los de mayor peso: el verde oscuro y el verde claro, que representan las dos respuestas más elevadas dentro de las siete posibles, lo que fue interpretado como que los expertos hicieron una valoración muy positiva del sistema.

Sistema de formación continua para la alfabetización digital de los docentes de las escuelas de hotelería y turismo cubanas. Raidell Avello, Raúl López y Raúl Alpizar. 
Dentro de estos resultados, la pregunta con una valoración menos favorecida fue la cinco (¿Posibilidad de emplearlo en otros contextos?) donde predomina el color verde claro, pero hay que tener en cuenta que es el segundo en importancia según la escala utilizada. Esto debe estar dado porque aunque el sistema puede ser adaptado a otros sistemas, indudablemente, en su concepción se tuvieron en cuenta particularidades de las EHT.

Las preguntas uno, dos, tres y cuatro son las que muestran una prevalencia del color verde oscuro, con el que se cubre gran parte del semiplano superior, donde se muestran el consenso favorable de los expertos en los temas que se trataron, mientras que en el semiplano inferior, predomina el color verde claro a pesar de existir áreas de color verde oscuro.

Lo antes expuesto evidencia un consenso casi unánime entre los expertos al emitir sus valoraciones de la preguntas uno, dos, tres y cuatro, un matiz más heterogéneo se evidencia en la valoración que dan a las pregunta cinco.

Al hacer el análisis por fila, o por expertos, se puede observar que solo dos tienen tres o más calificaciones con el color verde claro que, a pesar de no ser mala, no le otorgan la máxima calificación a las preguntas.

A partir de los análisis efectuados anteriormente se pueden hacer varias interpretaciones:

- Los expertos proporcionaron una alta puntuación a las preguntas, sin embargo, existió diversidad de criterios en las respuestas, que aunque altas, no fueron la máxima para todas las interrogantes, esto indica la seriedad y rigor en las respuestas de los expertos.

- La última pregunta, ¿Posibilidad de emplearlo en otros contextos?, fue la de menor valoración, porque los expertos, por su experiencia en el área, aprecian la debilidad que posee el sistema para aplicarse en otros escenarios, puesto que precisamente esta es una de sus particularidades, que aprovecha el contexto donde se desarrolla, aunque también expresaron que como todo sistema tiene posibilidades de adaptación.

Los expertos expresaron otras opiniones, no solo las colorimétricas solicitadas, sino otras plasmadas de forma literal, que indican valoraciones a favor del sistema de formación continua que se ha valorado. De manera general y sintética se destacan:

- Reúne las características generales de los sistemas reales (totalidad, centralización, jerarquización, integridad), con una intencionalidad bien definida y un grado de terminación adecuado, teniendo en cuenta de que todo sistema, en su dinámica natural evoluciona, se reestructura y modifica buscando la el perfeccionamiento continuo.

- Se evidencian los límites que lo definen como sistema y representa analíticamente la formación continua de los docentes en TIC, lo cual garantiza su posibilidad real de creación.

Sistema de formación continua para la alfabetización digital de los docentes de las escuelas de hotelería y turismo cubanas. Raidell Avello, Raúl López y Raúl Alpizar. 
- La calidad de la estructura: en este sentido los expertos expresaron que la manera que se organizó y estructuró el sistema es adecuado, ya que permite comprender mejor como enfrentar la formación de los docentes en TIC de manera sistémica.

- Con respecto al trabajo en subsistemas, expresaron que se expresan claramente las relaciones entre cada uno de ellos, además se muestra coherencia en los componentes que los componen.

- En relación al enfoque colaborativo del sistema, consideran que está bien planteado y expresado de manera clara en cada una de las formas de superación que se diseñaron.

- Asimismo, comentaron sobre la interactividad que provoca la estructura entre los componentes, brindándole el dinamismo y retroalimentación entre los componentes, necesarios para lograr la flexibilidad y adaptabilidad del sistema frente a los cambios del contexto y necesidades del sector turístico.

\section{Aplicación en la práctica. Ejemplo de tutoría colaborativa.}

Luego de valorado el sistema por los expertos, se inició su aplicación en la práctica en la EHT de Cienfuegos, en el 2013, con el objetivo de elevar el nivel de alfabetización digital de los 28 docentes de esta escuela. Se implementaron cursos, entrenamientos, tutorías colaborativas y talleres (Avello, Martín, Díaz, y Clavero, 2013), así como blogs docentes, biblioteca virtual, centro de recursos, entre otros. Con el objetivo de ilustrar alguna de estas acciones, a continuación se relata una tutoría colaborativa realizada.

La tutoría, definida como forma organizativa del proceso pedagógico profesional, en el sistema de EHT, tiene como objetivo fundamental que los estudiantes (en este caso los docentes) a través de la orientación y supervisión de un profesor, o instructor, desarrollen sus competencias profesionales en su vínculo directo con la entidad productiva. Consiste en un proceso en que el profesor guía al estudiante en la realización de un trabajo específico para solucionar problemas. La tutoría colaborativa, concebida en el sistema que se propone, comprende, además de los elementos anteriores, la colaboración entre los involucrados como estudiantes (docentes) en el sistema de formación.

La tutoría colaborativa que se comenta, se realizó entre dos docentes del departamento de Idiomas, específicamente entre el docente de idioma italiano a la profesora de idioma francés, en el curso 2013-2014. Esta, tuvo como objetivo transferir la experiencia práctica que tenía el docente de idioma italiano con respecto a la elaboración de libros electrónicos y fue coordinada por el profesor de informática que no tenía los conocimientos de esta aplicación, pero si conocía que el profesor de idioma había trabajado con este programa.

Los libro electrónicos, también conocidos como e-book, ecolibro o libro digital, es una versión electrónica o digital de un libro, es una publicación cuyo soporte no es el papel sino un archivo electrónico, su texto se presenta en formato digital y se almacena en disco, CD-ROM o en línea (en la Web), permite incorporar elementos multimedia como 
vídeo, audio, y en el caso de Internet, posibilita enlaces a otras páginas. Dentro de las aplicaciones para la creación de libros electrónicos se encuentra LIM, que es un entorno para la creación de materiales educativos, formado por un editor de actividades (EDILIM), un visualizador (LIM) y un archivo en formato XML (libro) que define las propiedades del libro y de cada una de las páginas.

La competencia a desarrollar, con respecto al marco de competencias propuesto por Avello y López, 2015, fue: Elabora páginas web y presentaciones multimedia. Como resultado, se logró diseñar un libro de ejercicios para enriquecer el aprendizaje del idioma francés en la esfera de servicio gastronómico, utilizando la herramienta informática EDILIM (Sánchez y Avello, 2014).

El libro electrónico cuenta con varias páginas de información general, así como 15 ejercicios y se nombró: El restaurante. Las primeras páginas, de información general, están dedicadas a contenidos instructivos del idioma específico (ilustraciones explicativas, diálogos, gramática, etc.) y luego los ejercicios, a continuación se puede observar una imagen ilustrativa del libro.

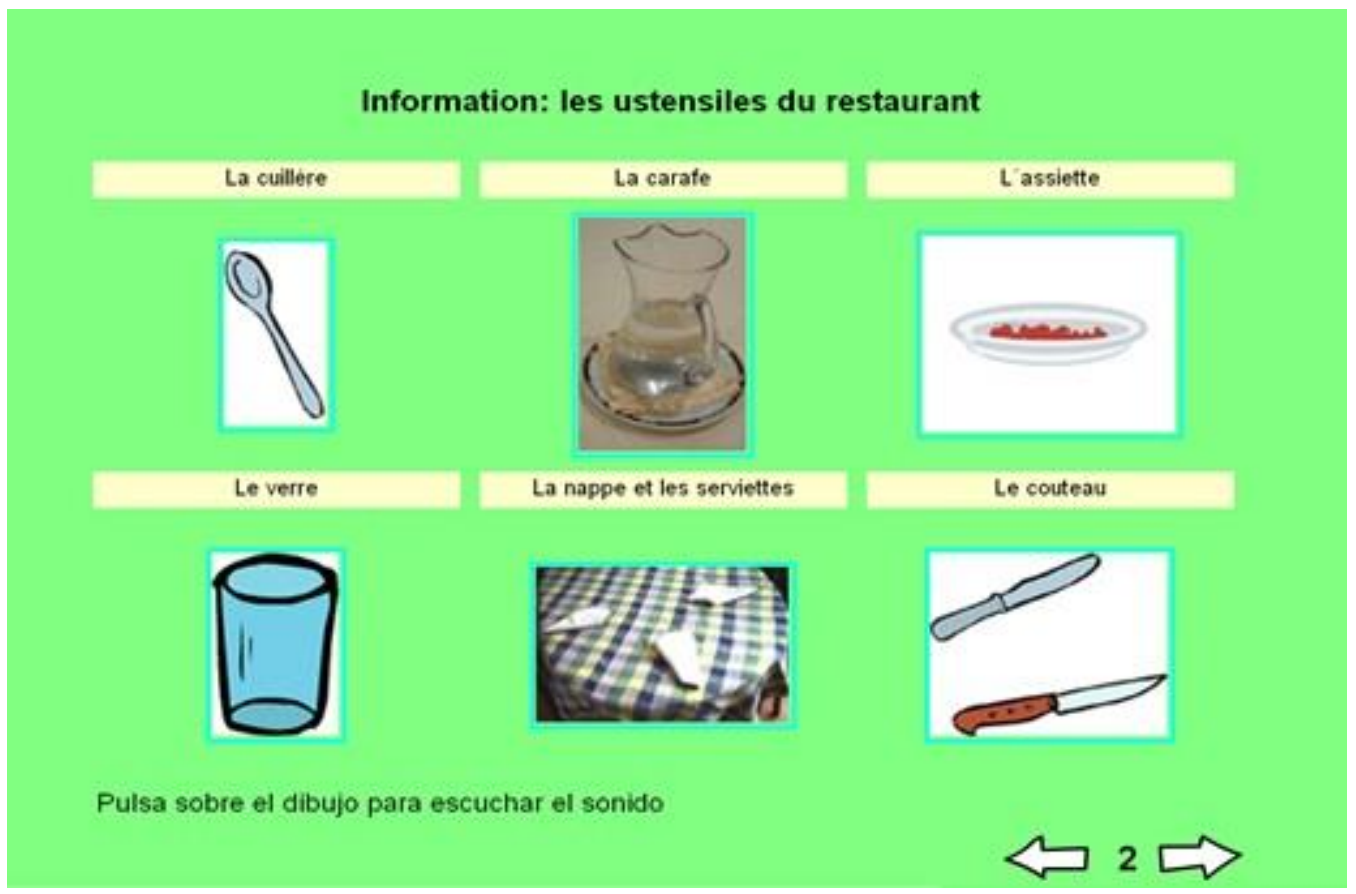

Figura 2. Imagen de una página del libro electrónico "El restaurante".

Adicionalmente se colocaron en la biblioteca los recursos (manuales y videotutoriales) encontrados y que sirvieron de apoyo en el proceso de autopreparación y tutoría de los docentes implicados. Además, expresaron que se sintieron muy satisfechos con esta forma de aprendizaje y se mostraron dispuestos a seguir replicando estas competencias desarrolladas a los docentes que expresaran su interés.

\section{Discusión general de la aplicación del sistema}


La valoración de los resultados obtenidos, hasta fines de 2015, pues el sistema se encuentra en aplicación, permitió corroborar las posibilidades que ofreció la formación continua de los docentes implementada y revelar los aspectos a considerar en la propuesta definitiva que se diseñó, como aporte de esta investigación, en función de la alfabetización digital de los docentes. Así, los participantes consideraron que las formas de superación recibidas y la manera en que se organizó el proceso propiciaron un uso inteligente de las TIC en los procesos sustantivos de las escuelas de turismo cubanas, y por las propias actividades de superación. Esto se evidenció en la frecuencia con que accedían a los materiales disponibles y consultas acerca del trabajo realizado, así como el incremento de sus expectativas de aprendizaje.

En cuanto a los cambios ocurridos en el orden socioprofesional, se pudo constatar, mediante la observación y entrevistas grupales realizadas, que los docentes se fueron interesando y solicitaron matricularse en las formas de superación o solicitar tutorías. En este proceso influyeron los cambios favorables en la motivación del grupo de docentes participantes, que comenzaron a tener resultados favorables con la aplicación de las TIC en su entorno de actuación, tanto en el aula como en los escenarios de educación e investigación en el trabajo.

Con respecto al enfoque colaborativo que se persiguió en la implementación del sistema, se aprovechó gran parte de la experiencia acumulada por los docentes que participaron en esta formación, lo cual permitió un amplio intercambio de ideas y reflexiones sobre las temáticas que se abordaron.

Por otra parte, las acciones de motivación, familiarización y de intencionalidad durante las formas de superación, propiciaron una mejor comprensión de las potencialidades de las TIC, aspecto que se hizo evidente en temas tan complejos como la estadística del turismo, simulación de procesos hoteleros, recepción hotelera, entre otros.

Se entiende que la formación produjo satisfacción al profesorado y que ésta fue de ayuda para la resolución de problemas en su desempeño. Por ello, en ningún caso debería plantearse la formación como normativa o amenaza que pudiese generar reacciones defensivas y de rechazo.

A partir de los resultados en la formación del profesorado se puede sugerir la utilización de metodologías que ayuden y faciliten el aprendizaje a través de la acción, la reflexión de la propia praxis, la autocrítica formativa $\mathrm{y}$, que a su vez promuevan el interés continuo de los docentes. Es especialmente importante un feedback, como ocurrió con el blog implementado, posterior de los resultados, una vez que los docentes se han formado, para poder analizar si la formación ha dado algún resultado o ha incidido de manera positiva en el modelo inicial planteado.

Hay competencias que fueron desarrolladas por los docentes solo con la autopreparación, con el apoyo de los recursos que se dispusieron en los servicios de información de la escuela, como el blog docente y las bibliotecas digitales, entre otros.

Es importante destacar que la preparación de los docentes se realizó según prioridades de su área, en decisión conjunta con su jefe de departamento, porque en algunas de ellas las prioridades de formación cambian, por lo que cada docente asumió un camino

Sistema de formación continua para la alfabetización digital de los docentes de las escuelas de hotelería y turismo cubanas. Raidell Avello, Raúl López y Raúl Alpizar.

Página 11 de 13 
diferente para su alfabetización digital, que indudablemente todavía está en proceso. Esto es evidente por el carácter sistémico de la alfabetización digital, sujeta a nuevas necesidades en continuo surgimiento.

Se identificó que los docentes que habían participado en la mayoría de las acciones formativas implementadas, pudieron incluso desarrollar actividades más complejas: funcionar como tutores de trabajos presentados en el Fórum Estudiantil de la EHT de Cienfuegos y en trabajos relativos a la aplicación de las TIC para la solución de problemas del turismo en el territorio. Esto evidenció los conocimientos y las motivaciones que respecto al tema se formaron en esos docentes, en los espacios no áulicos, aspectos que amplían la connotación de la alfabetización digital, tal y como se promueve y concibe en esta investigación.

\section{Conclusiones}

Se elaboró un sistema de formación continua en alfabetización digital para los docentes de las EHT compuesto por tres subsistemas interrelacionados entre sí, como también ocurre con los componentes que los integran. Es importante destacar el enfoque colaborativo del sistema, le concede una particularidad importante para el desarrollo colaborativo de las competencias necesarias en los docentes de la EHT para enfrentar las nuevas exigencias de la sociedad digital.

Es sistema propuesto fue valorado positivamente por los expertos por su estructura en subsistemas y la relación de los componentes que los integran, así como por la posibilidad de ser empleado en otros contextos.

De igual forma, los docentes, luego de las primeras acciones formativas, consideran acertado el sistema y opinan favorablemente sobre la forma de implementación de estas, así como los resultados obtenidos en el desarrollo de las competencias abordadas.

Presentación del artículo: 22 de marzo de 2016 Fecha de aprobación: 29 de marzo de 2016 Fecha de publicación: 30 de abril de 2016

Avello, R., López, R. y Alpizar, R. (2016). Sistema de formación continua para la alfabetización digital de los docentes de las escuelas de hotelería y turismo cubanas. RED. Revista de Educación a Distancia. 49(21). Consultado el (dd/mm/aaaa) en http://www.um.es/ead/red/49

\section{Bibliografía}

Area, M. (2009). El proceso de integración y uso pedagógico de las TIC en los centros educativos. Un estudio de casos. Revista de Educación, (352), 77-97. Recuperado de http://www.revistaeducacion.educacion.es/re352/re35204.pdf

Avello, R, y López, R. (2015). Alfabetización digital de los docentes de las escuelas de hotelería y turismo cubanas. Experiencias en su implementación. RUSC. Universities and Knowledge Society Journal, 12(3), 1-13. doi:10.7238/rusc.v12i3.1994

Sistema de formación continua para la alfabetización digital de los docentes de las escuelas de hotelería y turismo cubanas. Raidell Avello, Raúl López y Raúl Alpizar.

Página 12 de 13 
Avello, R., López, R., Gómez, M., Espinosa, G., \& Vázquez, S. (2014). Necesidades de formación en TIC de los docentes de las escuelas de hotelería y turismo cubanas. International Journal of Educational Research and Innovation, 1(2), 15-28. Recuperado de http://www.upo.es/revistas/index.php/IJERI/article/view/1065

Avello, R., Martín, I., Díaz, M. y Clavero, M. I. (2013). Zotero, más allá de un gestor bibliográfio. Una experiencia con los docentes y nuevas metas. Revista Didáctica, $\begin{array}{lllll}\text { Innovación } & y & \text { Multimedia, } & \text { Recuperado de }\end{array}$ http://dim.pangea.org/revistaDIM25/revista25ARzotero.htm

Crespo Borges, Tomás. (2009). Métodos de la Prospectiva en la Investigación pedagógica. La Habana: Educación Cubana.

Godet, M., y Durante, P. (2007). Prospectiva Estratégica: problemas y métodos (2ed). Paris: Prospektiker, Instituto Europeo de Prospectiva y Estrategia. Recuperado de http://www.prospektiker.es/prospectiva/caja-herramientas-2007.pdf

Gutiérrez, A. (2008). Las TIC en la formación del maestro. «Realfabetización» digital del profesorado. Revista Interuniversitaria de Formación de Profesorado, 22(3), 191-206.

López, R. (2009, Noviembre). El análisis de correspondencia. Una solución al Método Delphi. Congreso COMPUMAT 2009. UCP “Enrique José Varona”. La Habana.

Ministerio de Educación Superior (2004). Reglamento Educación de Posgrado. Resolución No. 132/2004. La Habana: Autor.

Novoa, B. M. (2006). Modelo pedagógico para la potenciación del liderazgo en directivos en las escuelas de hotelería y turismo (tesis de doctorado). Villa Clara, Cuba: UCP "Félix Varela".

Ortega, A., y Bracho, C. (2008). Prospectiva de las Tecnologías de Información y Comunicación: La explotación racional de informaciones tecnológicas. Multic, 8(2).

Salinas, J., de Benito, B., \& Lizana, A. (2014). Competencias docentes para los nuevos escenarios de aprendizaje. Revista Interuniversitaria de Formación del Profesorado, 28(1), 145-163. Recuperado de http://www.redalyc.org/articulo.oa?id=27431190010

Sánchez, M. y Avello, R. (2014). Libro electrónico interactivo de apoyo a la impartición del francés para servicio gastronómico. Revista Didáctica, Innovación y Multimedia, 29. Recuperado de http://dim.pangea.org/revistaDIM29/revista29OChosteleria.htm 